\title{
Contact X-ray microscopy of living cells by using LiF crystal as imaging detector
}

\author{
L. REALE*, F. BONFIGLI $\dagger$, A. LAI $\dagger$, F. FLORA $\dagger$, P. ALBERTANO $\ddagger$, M. L. DI GIORGIO§, L. MEZI $\dagger$, \\ R.M. MONTEREALI $\dagger$, A. FAENOV\|, T. PIKUZ\#, S. ALMAVIVA $\dagger$, M. FRANCUCCI $\dagger$, P. GAUDIO**, \\ S. MARTELLUCCI**, M. RICHETTA** \& A. POMA \\ *Campus Biomedico, Via Álvaro del Portillo 21, Rome, Italy \\ $\dagger$ †NEA, C.R. Frascati, UTAPRAD, Technical Unit Development and Applications of Radiation, V. Enrico Fermi 45, Frascati, Rome, Italy \\ $\ddagger$ Università di Roma “Tor Vergata,” Laboratorio di Biologia delle Alghe, Dipartimento di Biologia, via della Ricerca scientifica, Rome, Italy \\ §University of L'Aquila, Department of Life, Health and Environmental Sciences, Coppito, L'Aquila, Italy \\ |Institute for Academic Initiatives, Osaka University, Japan and Joint Institute for High Temperatures Russian Academy of Sciences, Moscow, Russia \\ \#Graduate School of Engineering, Osaka University, Japan and Joint Institute for High Temperatures Russian Academy of Sciences, Moscow, Russia \\ ** University of Rome "Tor Vergata,", Department of Engineering, Via del Politecnico 1, Rome, Italy
}

Key words. Contact X-ray microscopy, lithium fluoride detectors, algae,

Chlamydomonas, Leptolyngbya, Macrophage RAW 264.7.

\section{Summary}

In this paper, the use of lithium fluoride (LiF) as imaging radiation detector to analyse living cells by single-shot soft $\mathrm{X}$-ray contact microscopy is presented. High resolved X-ray images on LiF of cyanobacterium Leptolyngbya VRUC135, two unicellular microalgae of the genus Chlamydomonas and mouse macrophage cells (line RAW 264.7) have been obtained utilizing $\mathrm{X}$-ray radiation in the water window energy range from a laser plasma source. The used method is based on loading of the samples, the cell suspension, in a special holder where they are in close contact with a LiF crystal solid-state Xray imaging detector. After exposure and sample removal, the images stored in LiF by the soft X-ray contact microscopy technique are read by an optical microscope in fluorescence mode. The clear image of the mucilaginous sheath the structure of the filamentous Leptolyngbya and the visible nucleolus in the macrophage cells image, are noteworthiness results. The peculiarities of the used X-ray radiation and of the LiF imaging detector allow obtaining images in absorption contrast revealing the internal structures of the investigated samples at high spatial resolution. Moreover, the wide dynamic range of the LiF imaging detector contributes to obtain high-quality images. In particular, we demonstrate that this peculiar characteristic of LiF detector allows enhancing the contrast and reveal details even when they were obscured by a nonuniform stray light.

Correspondence to: Francesca Bonfigli, ENEA, C.R. Frascati, UTAPRAD, Technical Unit Development and Applications of Radiation, V. Enrico Fermi 45, 00044 Frascati, Rome, Italy. Tel: +39-0694005567; fax: +39-0694005400; e-mail: francesca.bonfigli@enea.it

\section{Introduction}

The use of soft X-rays (300 eV $<h v<10 \mathrm{keV}$ ) for imaging investigations in life science has great perspective thanks to the peculiar properties of this radiation. The wavelength values in this spectral region allow a very high spatial resolution, few nanometres and imaging of internal features of the biological sample is possible thanks to the X-ray pathways inside the sample itself.

The great and fast development of X-ray sources, optics and detectors, allow using it for many applications in different fields, by means of several microscopy techniques (contact, projection, full field and scanning microscopy) and operation modes (absorption contrast, phase contrast, tomography, etc.; Chao et al., 2005; Sakdinawat \& Attwood, 2010).

The projection microscopy configurations need optics (Fresnel Zone Plates) to focus radiation on the sample and to project a magnified image of the object onto detectors that can possess low spatial resolution, such as charge-coupled device (CCD) camera (Martz et al., 2012). The use of Fresnel Zone Plates optics requires monochromatic radiation, with a consequent reduction of the photon flux on the samples. This means long exposure times and need of cooling the biological specimens at cryogenic temperature, making impossible dynamic studies and in vivo observations. Holographic techniques need coherent radiation. At synchrotron facilities, a spatial filtering is needed to select the coherent part of the beam with a consequent loss of intensity and long exposure times. Coherence, monochromaticity and high brillance of an X-ray free electron laser (Ackermann et al., 2007) will allow obtaining of images with very high spatial resolution of biological samples in coherent diffractive configurations (Chapman et al., 2006). In 


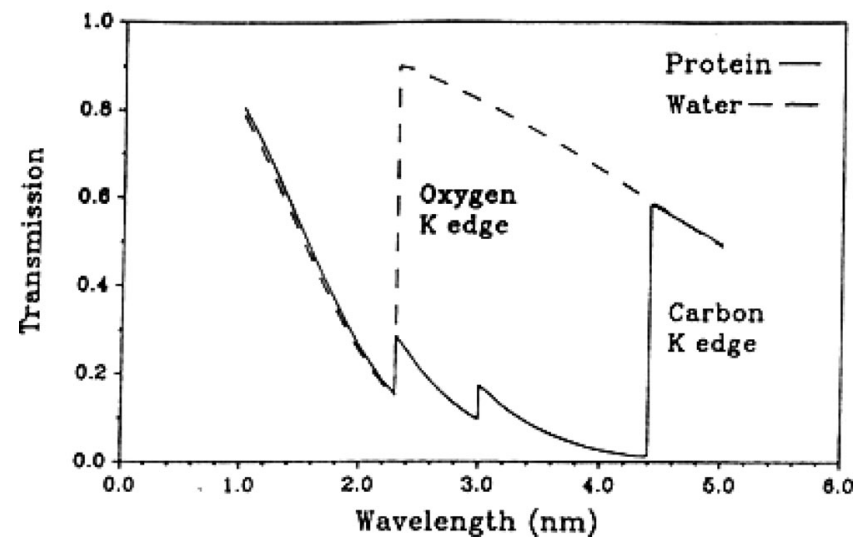

Fig. 1. Transmission versus wavelength of water and proteins for $1 \mu \mathrm{m}$ thickness.

this case, the effect of X-ray-induced sample damage on the recorded image or diffraction pattern is relevant and it could be reduced, if we could collect diffraction data faster than the relevant damage processes (Neutze et al., 2000).

$\mathrm{X}$-ray contact microscopy represents the simplest imaging technique: the sample is placed in direct contact with a sensitive material and exposed to radiation (Cotton et al. (1995)). The image is formed in an absorption contrast process. This process does not need coherent radiation and compact polychromatic laboratory X-ray sources, such as soft X-ray laser plasma sources, can be used. Single-shot experiments are possible in this experimental configuration, allowing in vivo investigation (without the need of any cryogenic or chemical process). The spatial resolution of the images obtained by contact X-ray microscopy is limited by penumbra blurring effects, diffraction effects and by the spatial resolution of the detector. The drawbacks of standard detectors, mainly in terms of spatial resolution, have limited the use and the development of this simple laboratory technique. The performances of LiF detectors have renewed the interest in such experiments and for the development of X-ray imaging laboratory tools.

In the soft X-rays interval there is a narrow energy range between the carbon and oxygen absorption K-edges ( $h v=$ $284-532 \mathrm{eV}$, or wavelengths $4.4-2.3 \mathrm{~nm}$ ), named the water window, where water is rather transparent to the X-rays whereas proteins or other carbon components are not (Fig. 1).

This behaviour creates a natural absorption contrast in the image of in vivo cells even without any contrast enhancement treatment. Moreover, the penetration length of X-rays inside the sample at the energy values of the water window is very close to the typical thickness of the cell itself, thus allowing to image full and alive cells. No other imaging techniques allow going through the structure of an alive cell at a resolution better than that of optical microscopes. For example, other well-known microscopy techniques, like scanning electron microscopy (SEM) and transmission electron microscopy
(TEM), need special chemical treatment of the sample to create a contrast in the sample structures. In these methods, the cell undergoes fixation, dehydration, embedding, sectioning and dying with different electron dense substances (osmium tetraoxide, lead citrate, uranyl acetate, etc.; Reynolds 1963). All such treatments necessarily change the real status of living cells, mostly from the point of view of the chemical composition (Larabell \& Le Gros, 2004), and imaging results because of the possibility to introduce artefacts. Even cryo-sectioning of frozen specimens and subsequent microscopy observation will allow investigations of dead cells only.

In this article, the results of soft X-ray contact imaging experiments in the water window range of (1) Leptolyngbya sp. VRUC 135, (2) Chlamydomonas reinhardtii and C. dysosmos and (3) animal cells of macrophage line RAW 264.7 obtained by an innovative LiF imaging radiation detector, based on the stable formation of photoluminescent colour centres (CCs), are presented.

The obtained images show the external morphology and internal features of the above mentioned three different types of biological samples, thus demonstrating the applicability of this imaging technique either to plant or animal cell line in biology studies.

This study could extend the field of interest of SXCM to utilize the technique for morphological analyses to identify cell types and structures, phase of cell cycle and other subcellular features suitable for biological and medical studies.

More than 10 years ago, the high spatial resolution imaging detector used for SXCM technique was typically polymethyl methacrylate (PMMA) photoresist (generally used in microelectronics). The PMMA molecules change their properties when hit by X-rays and this causes a different development behaviour in the presence of the developer and fixer solutions. After development, the relief map of a sample was originated on the surface of the PMMA resist and then observed with atomic force microscopy (AFM) by scanning the resist surface, which results in a digital image of the sample structure. Another possibility was the observation of the resist after evaporating of a metal on its surface for SEM observation. X-ray microradiographies obtained on PMMA imaging detector are shown in the following. PMMA imaging detector allows a resolution theoretically up to the dimension of the PMMA molecule (a few nanometres). However, the effective resolution, about 50 $\mathrm{nm}$, depends on the read-out process of the images (chemical development plus AFM reading). Furthermore, the developing procedure was highly irreproducible, mainly because of the extremely poor dynamic range of PMMA, and this create great difficulties in the final analyses of the image. In practice, the relief image, which appears gradually on PMMA, changes a lot during development. Sequentially, it shows first the thinnest less X-ray absorbing structures of the cell and then the most $\mathrm{X}$-ray opaque ones. For this reason, during the development of PMMA not a single image is obtained but a sequence of images, which cannot be easily combined with each other. 
The disadvantages of the PMMA as imaging detector for SXCM, such as its poor dynamic range, the dependence of the image quality on the development process and the time consuming use of AFM, are overcame by novel X-ray imaging detectors based on optically active CCs in LiF [ENEA Patent TO2002A000575, G. Baldacchini, F. Bonfigli, A. Faenov, F. Flora, R.M. Montereali, A. Pace, T. Pikuz, L. Reale; International No.: WO 2004/005906 A1]. A detailed comparison of LiF with other soft X-ray detectors is reported in Baldacchini et al.(2005). These radiation imaging detectors showed a great range of applications and allowed observing cell structures (cell exudates, dry pollens, internal structures) not easily visible by other techniques (Bonfigli et al., 2003, 2005, 2008; Reale et al., 2008). The peculiarities of luminescent LiF detectors (Baldacchini et al., 2005), like high spatial resolution over a large field of view, wide dynamic range (i.e. a large number of grey levels), simplicity of use and efficiency of the optical reading technique, can be exploited for X-ray microscopy in different configurations, even for in vivo and lens-less imaging.

As demonstrated in the following, even when conventional optical fluorescence microscopes are used for LiF reading and some details are obscured by nonuniform stray light generated by the illumination source, still the high dynamic of LiF can be exploited to partly remove the stray light effects and to reconstruct the original image, which was directly stored in $\mathrm{LiF}$ by CCs induced by soft X-rays passing through the investigated samples.

\section{Material and methods}

\section{Biological characteristics of the imaged samples}

The imaged samples have different biological characteristics. They can be summarized as follow:

\section{(1) Cyanobacterium Leptolyngbya sp. VRUC 135}

Cyanobacteria are the first photo-oxygenic prokaryotes that colonized the earth surface in the early history of life on our planet. It is thanks to their photosynthetic activity that the oxygen and oxidative atmosphere appeared on the earth. They have special phycobilin pigments, allophycocyanin, phycocyanin and phycoerythrin present in the photosynthetic antenna complexes, and scytonemins in the sheath that allow the absorption of the UV dangerous radiation. This sample is a subaerophytic epilithic filamentous cyanobacterium growing typically on wall paintings in subterranean archaeological sites. This species is adapted to extremely low light conditions and is able to perform phototaxis and to acclimate to different light irradiance and wavelengths in the visible range (Albertano \& Caiola, 1988a;b; Albertano, 1991; Bruno \& Albertano, 1999; Albertano et al., 2000). The very thin trichome ( $<3 \mu \mathrm{m}$ in diameter) is surrounded by a colourless sheath open at the ends that is mostly made of hetero-polysaccharides (Bellezza et al., 2003).

\section{(2) Chlamydomonas}

Chlamydomonas is a genus of green microalgae that have been used to study different aspect of evolutionary biology and ecology. The fact that most representatives of this taxon have a short generation time, can be facultatively autotrophic and heterotrophic and reproduce both sexually and asexually along with lot of genetic information available for some species has made these organisms of choice for many selection experiments (Oldenhof et al., 2006; Renaut et al., 2006). More specifically, Chlamydomonas reinhardtii and Chlamydomonas dysosmos are motile unicellular green algae found commonly in soil and freshwater, 5-15 $\mu \mathrm{m}$ in diameter, that swim with two apical flagella. The cell wall is composed of hydroxyproline-rich glycoproteins. In the apical region, an orange eyespot sensible to light is present inside the chloroplast, which has the shape of a large cup. The interest in green microalgae recently increased because of their application in the removal of heavy metals. For example, two experimental co-incubation systems have shown that the use of $C$. reinhardtii can reduce the genotoxic potential of heavy metals on vascular plants (Hordeum vulgare, Pisum sativum).

\section{(3) Mouse macrophages, cell line RAW 264.7}

The mouse macrophages, cell line RAW 264.7 is the leukaemia virus-transformed mouse macrophage cell line, RAW 264.7 (ATCC TIB-71). Macrophages are part of the innate immune system, recognizing, engulfing and destroying many potential pathogens and can also recognize syngeneic tumour cells and some virus-infected cells as well as normal cells undergoing programmed cell death (apoptosis). Mononuclear phagocytes are present in every organ in the body as a major cell population. The ability of the blood monocyte to take on so many different phenotypic states suggests that they may be able to act as a kind of mobile stem cell giving rise to many other cell types (Van Furth et al., 1972, Athanasou (1995), Banchereau \& Steinman (1998), Hume \& Gordon (1983), Hume et al. (1984a), Hume et al. (1984b), Hume et al. (1987), Larsen et al. (2002), Laskin et al. (2001), Lee et al. (1985), LohmannMatthes et al. (1994), Udagawa et al. (1990).).

\section{Characteristics of the X-ray source}

A high-power Nd: YAG/Glass laser was used to obtain a laser plasma X-ray source suitable for soft X-ray contact microscopy of biological samples developed at the University of Rome "Tor Vergata” (Petrocelli et al., 1993a, Petrocelli et al., 1993b; Bellucci et al., 2000; Richetta et al., 2006). The Nd: YAG/Glass laser emits a maximum pulse energy of about $10 \mathrm{~J}$ and has a pulse duration of 15 nanoseconds at the wavelength of $\lambda=1064 \mathrm{~nm}$. The laser beam is focused 


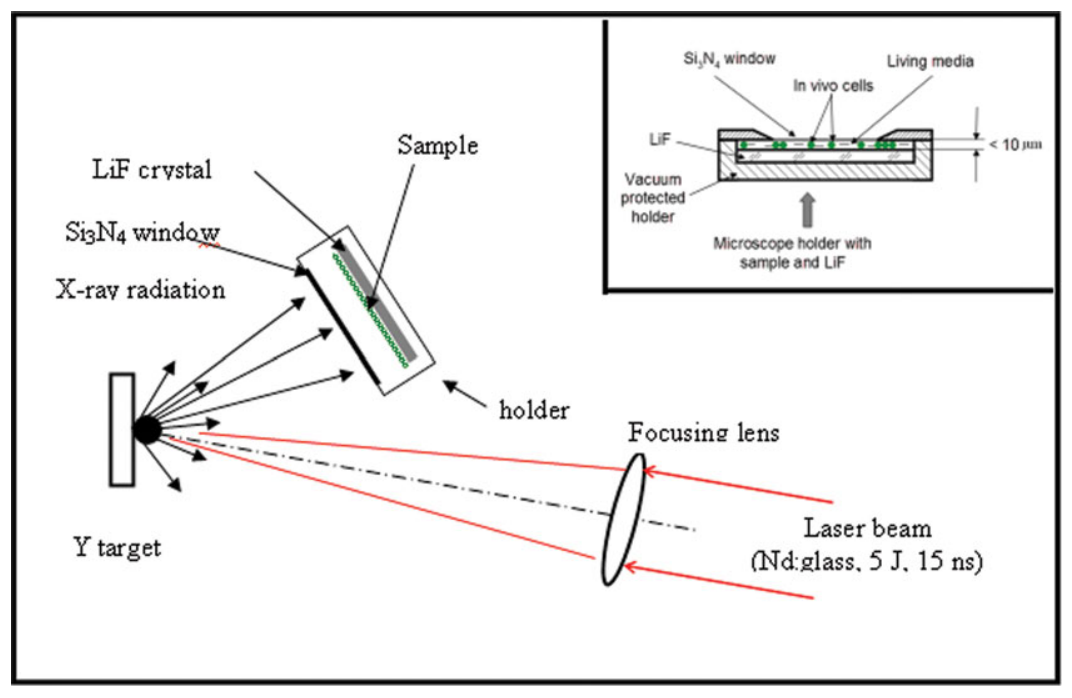

Fig. 2. Schematic of the experimental apparatus used for SXCM. The inset reports the microscope holder used for in vivo single-shot imaging experiment of living cells on LiF detectors.

on a tape target of the Yttrium material by a $f^{\#}=15$ doublet lens. Generally $f^{\#}$ is the ratio between the lens focal length and the lens diameter, but in our case we mean the ratio between the lens focal length $(30 \mathrm{~cm})$ and the laser beam diameter at the lens (which is smaller than the lens diameter). The target is placed in the middle of a vacuum chamber. A scheme of the experimental layout for contact microscopy is shown in Figure 2. The living media with cells, placed in contact with the LiF crystal surface, were protected from the vacuum by a special microscope holder (in the following called 'X-ray microscope'), with a silicon nitride $\left(\mathrm{Si}_{3} \mathrm{~N}_{4}\right)$ window just $100 \mathrm{~nm}$ thick (see inset Fig. 2). The sample is positioned at a short distance $(\sim 8 \mathrm{~mm})$ from the source in order to collect more photons in a single shot. Yttrium is a good emitter in the water window for microscopy experiments (Albertano et al., 1998); the X-ray pulse energy emitted in the full $2 \pi s r$ in the water window spectral region by using this source is about $0.4 \mathrm{~J}$, corresponding to an estimated conversion efficiency (in the water window spectral band) of 5\%. The X-ray source diameter is rather large $(\Phi \sim 300 \mu \mathrm{m})$ because of the low numerical aperture (NA $\sim 0.03$ ) of the focusing lens.

In lensless imaging in absorption contrast, the spatial resolution is also limited by the combination of three effects: source size, detector resolution, and diffraction effects. The relatively large size of the source and sample thickness (Wachulak et al., 2011) could give some problems of penumbra blurring. For the in vivo X-ray contact microscopy experiments (imaging of alive single cells in water suspension and by a single laser shot), the sample is positioned just at $d=8 \mathrm{~mm}$ from the source in order to collect a sufficient photon density in a single shot; in these conditions, for a typical gap $G=1-3$ $\mu \mathrm{m}$ between the LiF detector exposed surface and the internal structures of the sample, the penumbra blurring $\delta=\Phi \times \frac{G}{d}$ is less than $100 \mathrm{~nm}$. In some cases, for thicker samples, a bit larger distance, between source and sample, is used as best compromise between the minimization of the penumbra and the achievement of a sufficient photon flux on the detector in a single shot.

Although for projection microscopy the X-ray beam shaping is widely used to optimize the coupling between the illumination of the sample and the projection optics, in the case of SXCM the advantages of beam shaping are unfortunately reduced by the Liouville's theorem for radiation that implies the conservation of the radiation brightness: the smaller the size of the virtual source generated by the beam shaping optics, the larger the beam divergence and, consequently, the smaller the fraction of radiation that reaches the detector. The same effect for penumbra reduction could be reached simply by increasing the sample to source distance.

Alternatively, a laser-plasma source based on an excimer laser has been used at the ENEA Frascati laboratories. In that case the laser wavelength is much shorter $(308 \mathrm{~nm})$, the pulse energy is lower ( $5 \mathrm{~J}$ ) and the conversion efficiency in the waterwindow is also lower, so that the X-ray pulse energy is one order of magnitude less (see Albertano et al., 1998), but the focusing optics has a much larger numerical aperture (NA = 0.3) so that the source size (and the penumbra blurring as well) is almost one order of magnitude smaller.

\section{Cell samples preparation}

The cells were grown in vitro in controlled culture condition and then exposed at the X-rays. The strains of Leptolyngbya sp. VRUC 135, C. reinhardtii and C. dysosmos were provided by the Laboratory of Biology of Algae of Tor Vergata University (Italy), and grown in liquid BG11 (Rippka et al., 1979) and 
BBM (Bold, 1949) mineral media respectively, at $18^{\circ} \mathrm{C}$, with a light:dark cycle of 14:10 hours, for about 2 weeks. Samples were collected by centrifugation and used for exposure.

The leukaemia virus-transformed mouse macrophage cell line, $R A W 264.7$ (ATCC TIB- 71 ) was a kind gift by Prof. M.G. Cifone, Department of Health Sciences, University of L'Aquila. Because macrophages are the primary responders to different particles that initiate and propagate inflammatory reactions and oxidative stress, in the past the same authors used RAW 264.7 as an in vitro model system for applications in dual-energy synchrotron microradiography for mapping heavy metals or particles accumulation inside cells. Like in that case, even now cells were cultured in $75 \mathrm{~cm}^{2}$ flasks with DMEM (Cat. No. D6171, Sigma-Aldrich, Milan, Italy) supplemented with $1 \%$ L-glutamine, $1 \%$ penicillin/streptomycin and $10 \%$ heat inactivated foetal bovine serum (Celbio). Culture flasks were stored in a humidified incubator at $37^{\circ} \mathrm{C}$ and $5 \%$ $\mathrm{CO}_{2}$. Passaging of cells was performed by washing twice in phosphate-buffered saline ( $150 \mathrm{~mm} \mathrm{NaCl}, 10 \mathrm{~mm} \mathrm{NaPO}_{4}, \mathrm{pH}$ 7.4), and then removed from the flasks with a cell scraper every 2 days. Cell counts were assessed using a hemocytometer. They are about $15 \mu \mathrm{m}$ in diameter.

In this paper only the SXCM images of these untreated sample are provided.

\section{LiF soft X-ray imaging detector}

Innovative high-performance soft X-ray imaging detector based on stable CCs in LiF was proposed few years ago for contact X-ray microscopy and micro-radiography (Baldacchini et al., 2005; Bonfigli et al., 2005; ENEA Patent TO2002A000575, G. Baldacchini, F. Bonfigli, A. Faenov, F. Flora, R.M. Montereali, A. Pace, T. Pikuz, L. Reale).

$\mathrm{LiF}$ is a radiation-sensitive material, well known in dosimetry and utilized in photonic light-emitting devices (Montereali, 2002). CCs can be produced in LiF crystals and films by different kinds of ionizing radiation, such as soft X-rays, hard Xrays, $\gamma$-rays, high-energy particles, etc. Different types of CCs are simultaneously generated in LiF when exposed to ionizing radiation. The primary F-centre, consisting of an anion vacancy occupied by an electron, is the simplest one and it plays a crucial role in the formation of more complex defects like, for example, $\mathrm{F}_{2}$ and $\mathrm{F}_{3}{ }^{+} \mathrm{CCs}$. These $\mathrm{CC}$ are $\mathrm{F}$-aggregates consisting of two electrons bound to two and three close anion vacancies, respectively, and are particularly interesting because they are optically active. $\mathrm{F}_{2}$ and $\mathrm{F}_{3}{ }^{+}$centres are both efficiently created in LiF by soft X-rays (Bonfigli et al., 2005), they have almost overlapping broad absorption bands peaked at around $450 \mathrm{~nm}$ and, therefore, can be simultaneously excited with a single optical source of blue light. When observed under a blue excitation light, $\mathrm{F}_{2}$ and $\mathrm{F}_{3}{ }^{+} \mathrm{CCs}$ absorb at this wavelength and emit photoluminescence at longer wavelengths, in the red and green spectral ranges, respectively, with an emission intensity locally proportional to their density, thus revealing the
X-ray two-dimensional intensity map on LiF and hence also the radiographic image of the biological sample, which was in contact with LiF during the exposure to X-rays. Other alkali halide crystals can host CCs, but CCs in LiF are stable at room temperature (Montereali, 2002).

As far as the needed X-ray fluence values are concerned, for $\mathrm{LiF}$ and for any other image detector a large X-ray fluence (up to $1 \mathrm{~J} \mathrm{~cm}^{-2}$ ) is required for high quality (i.e. high resolution) SXCM images, just because of the statistical noise effects, no matter what detector is used. In fact, the small detail of a biological object can be distinguished from the neighbouring tissue only when its shadow reduces the X-ray flux by an amount larger than the statistical fluctuations of the X-ray fluence transmitted by similar details. LiF well fits this requirement, because its saturation level is beyond $1 \mathrm{~J} \mathrm{~cm}^{-2}$ at the used Xray energies (Baldacchini et al., 2005). After the exposure to $\mathrm{X}$-rays, the LiF plate storing sample image is illuminated with blue light and the fluorescent CCs spatial distribution, which reproduces the absorption contrast image of the sample, is observed by an optical microscope in fluorescence mode. While using luminescent LiF as soft X-ray imaging detector, the spatial resolution is limited by the image reading optical process; as a matter of fact, the intrinsic spatial resolution of LiF detectors is, in principle, limited only from the dimension of the produced CCs (a few nanometres; Sekatski \& Letokhov, 1996). Anyway, by using as reading process of LiF luminescent detectors advanced optical microscopes, like confocal laser scanning microscope and scanning near-field optical microscope, optical fluorescence images with micro- and nanometric spatial resolutions can be respectively obtained (Almaviva et al., 2006; Ustione et al., 2006).

The processes of formation and stabilization of primary and aggregate colour centres in LiF crystals after irradiation have been investigated since long times. It was found that the kinetic of colouration is quite complex and strongly dependent on a large number of variables, that is type of ionizing radiation and its characteristics, conditions of irradiation, like radiation intensity (dose rate), as well as chemical purity of the crystal, etc.

The prediction of CCs behaviour is still more difficult at room temperature, because of the higher mobility of vacancies, and at high defect concentrations, because of interactions among different types of CCs, and careful experimental measurements should be carried out (Voitovich et al, 2013). In the case of soft X-ray irradiation of LiF crystals at room temperature in a laser-plasma source, very few data are available in the literature (Bonfigli et al., 2009). However, on the basis of available data about the interactions of $\mathrm{F}_{2}$ and $\mathrm{F}_{3}{ }^{+}$colour centres with other complex CCs in LiF, specially $\mathrm{F}_{2}{ }^{+}$(Ter-Mikirtychev \& Tsuboi, 1997), it can be inferred that a stable condition is reached 24-32 h after irradiation. In our case the fluorescence images were acquired well after this time interval and no appreciable change was experimentally observed in successive optical readouts of the 
cells X-ray microscopy images stored in LiF crystal detectors in the used conditions.

The use of LiF detectors for SXCM in the water-window offers great potentialities: high spatial resolution, wide dynamic range, an efficient and direct optical process to detect the stored luminescent images without any previous development process. Moreover, LiF imaging detectors based on CCs photoluminescence offer the possibility to store the images for long time (several years) and to look at them at any time (with different microscopes, different magnification, different field size, etc.) without degradation. The used crystals are commercially available plates of $(5 \times 5 \times 0.5) \mathrm{mm}^{3}$ in dimensions, polished on both surfaces.

In SCXM experiments on LiF crystal detectors, the reading process of the luminescent LiF-based X-ray microradiographies was performed by using a Leica optical microscope in fluorescence mode.

\section{Method for the sample exposure}

A drop of the cells suspension in their medium has been loaded inside a special sample holder, called 'the X-ray microscope', that keeps the cell suspension and the LiF imaging detector in contact each other, as described before. The thickness of water in the holder is regulated as thin as possible according to the cell thickness itself, usually it reaches about $10 \mu \mathrm{m}$. The water thickness adjustment is reached as an equilibrium between the force of a spring (not shown in Fig. 2), placed behind the $\mathrm{LiF}$ crystal, that pushes the crystal toward the $\mathrm{Si}_{3} \mathrm{~N}_{4}$ window and the opposite force given by the water surface tension effect.

In this way an unicellular monolayer of the cells in the water has been formed. The X-ray microscope holder settled four elements: a $\mathrm{Si}_{3} \mathrm{~N}_{4}$ window to separate the water suspension from the vacuum, the drop of the water suspension with the cells, the LiF crystal detector (all together in a sort of sandwich where the LiF crystal surface is in close contact with the cell samples), and an elastic screw, which pushes on the back of the LiF detector to adjust the water thickness. The $\mathrm{X}$-ray microscope holder is positioned at $8 \mathrm{~mm}$ from the Yttrium target in the vacuum chamber of the Nd-YAG pumped laser-plasma source. Before inserting the LiF crystal detector into the X-ray microscope, a benzene drop is let to fall over the crystal and slowly dry; in this way the residual fat layer, which forms from the evaporated benzene, protects the crystal surface from the soluble action of the water suspension. In fact, although $\mathrm{LiF}$ is almost not-hygroscopic, the aqueous medium after the exposure to X-rays could damage the very thin $(<100 \mathrm{~nm})$ layer containing the formed CCs, that is the layer storing the image of the biological sample. Immediately after the single-shot X-ray exposure, the silicon nitride window often gets broken because of the debris bombardment, with the cells dying and the solution evaporating into the vacuum, but this explosion does not damage LiF at all. Then, after

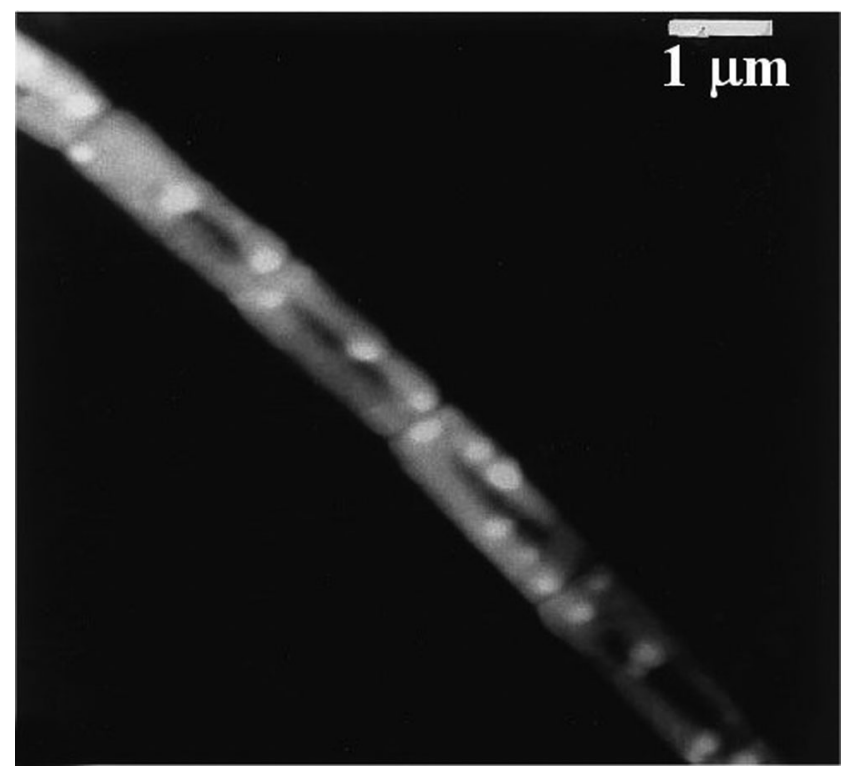

Fig. 3. Image of the cyano-bacterium Leptolyngbya V-ruc 155 (Albertano, 1997) obtained with the soft-X-ray contact technique on a resist of PMMA and observed with the atomic force microscopy (AFM).

air is let to refill the vacuum chamber, the LiF crystal is cleaned mechanically with great care using a cotton-stick by alcohol to remove the thin benzene layer together with the dead cell remnants.

\section{Results and discussion}

First of all, for comparison with the results obtained on $\mathrm{LiF}$ crystal detectors, two cells images obtained with the SXCM (in the water-window energy range) on PMMA photoresist are shown in Figs. 3 and 4, both obtained with the excimer laser plasma source of ENEA-Frascati on a resist of PMMA and observed at AFM. Both images have been already published in (Albertano et al., 1997). Figure 3 shows the images of Leptolyngbya sp. VRUC135. In this image one of the filaments typical for this cyanobacterium is well evidenced. As shown in the article, the image of the leptolyngbya changes a lot as a function of the PMMA developing time. Figure 4 shows the X-ray image of a $C$. dysosmos. This unicellular Chlorophyceaen green alga is usually characterized by the presence of two flagella apically inserted. Inside the cytoplasm, a large cup-shaped chloroplast with one pyrenoid surrounded by many starch granules, and the nucleous are visible in the image.

Moving to the images obtained on LiF crystals, Figure 5 shows the X-ray images of living $C$. reinhardtii. This species is bigger (around 10-15 $\mu \mathrm{m}$ in diameter) than the species C. dysosmos (about $5 \mu \mathrm{m}$ in diameter) previously shown in Figure 4, and its larger size allows to better look at internal structures. The image stored in the LiF crystal has been detected by a Leica optical microscope in fluorescence mode. 


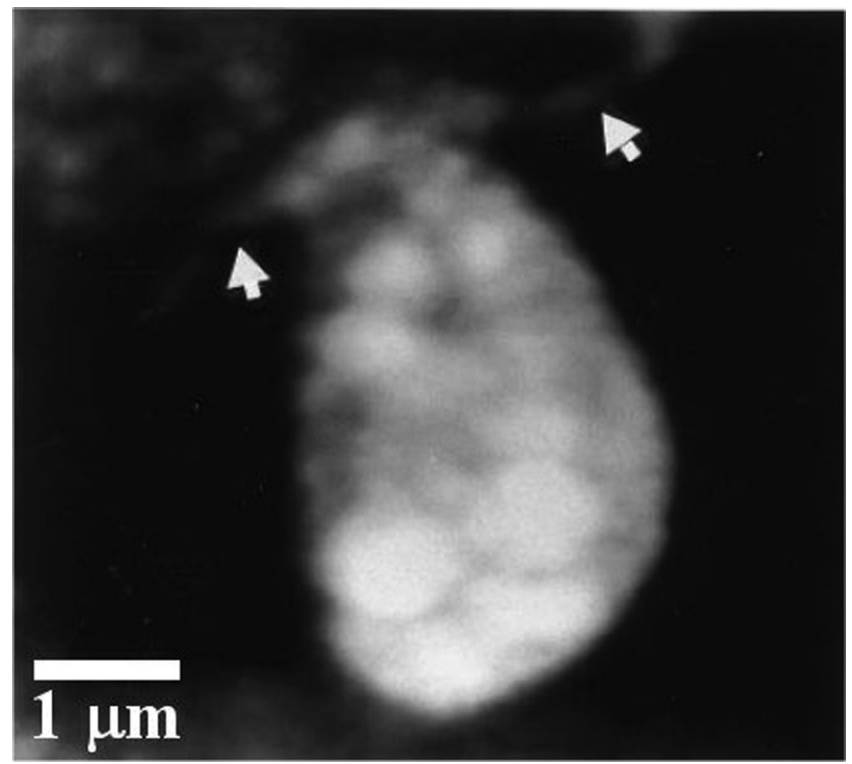

Fig. 4. Image of the alga Chlamydomonas dysosmos moewus (Albertano, 1997) obtained with the soft-X-ray contact technique on a resist of PMMA and observed with the atomic force microscopy (AFM). White arrow point out the two flagella of the cell.

The whole image shows three $C$. reinhardtii cells (zoomed in Fig. 5A-D) at amplified contrast (Fig. 5A and b refer to the same zone of the image just with different contrast). In Figure 5A), the nucleous (n) in the central area of the cell, and the pyrenoid (p) with several starch granules $(\mathrm{g})$ inside the peripheral chloroplast are well recognizable, for the first time on LiF X-ray imaging detector, by the different grey tones. The optical fluorescence microscope here used has not allowed seeing the two flagella apically inserted, because they are thinner than the microscope resolution $(\sim 400 \mathrm{~nm})$. The Figure 5(C and D) allow to recognize the same internal structures but the shape of the cells are not so well defined. In Figure 5(D) are well visible the starch granules, whereas in Figure $5(C)$ the large cup-shaped chloroplast is partially shown.

In Figure 6, the shape of the $C$. reinhardtii cell and sporangium (Fig. 6B), stored in a LiF crystal detector, is shown. The vegetative reproduction process is because of two mitotic events of cell division, which leads to the release of four autospores. Under ideal growth conditions, cells may undergo two or three rounds of mitosis before the daughter cells are released from the old cell wall into the medium. Thus, a single growth step may result in four or eight daughter cells per mother cell. In certain conditions the alga also undergoes sexual conjugation. Each autospore has a small chloroplast and one nucleous in close proximity of the new septa (Fig. 6B arrows) as it has been observed in several green algae (PickettHeaps, 1975). These organelles are visible because of their different X-ray absorption.

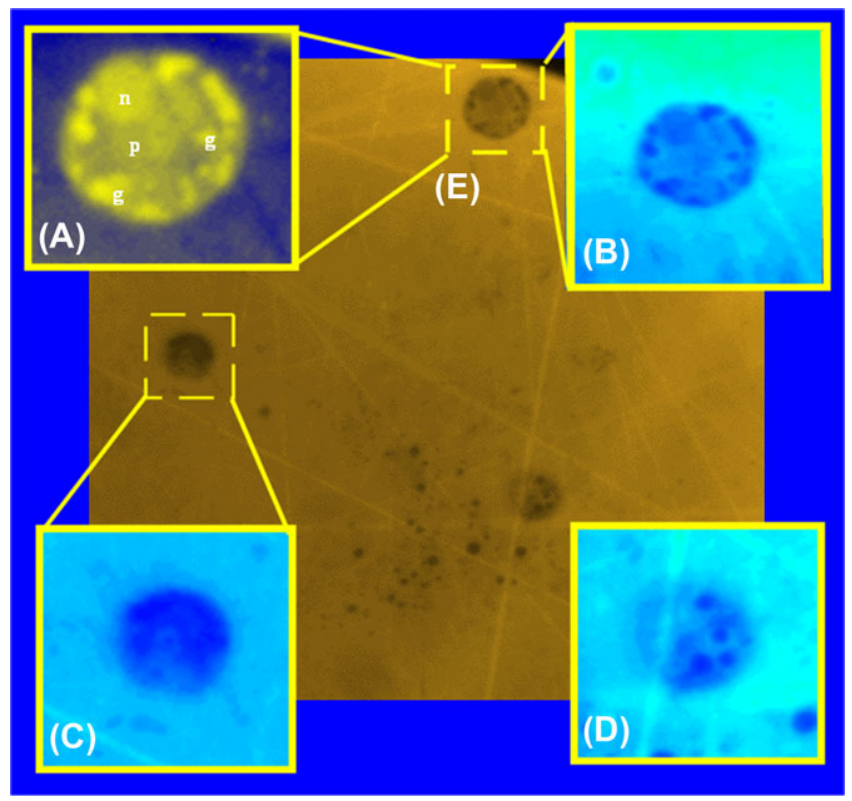

Fig. 5. X-ray images in the water-window of in vivo C. reinhardtii cells stored in a LiF crystal (exposed at the Nd-YAG laser plasma source) and observed by a fluorescence microscope. The whole image size is $140 \times 140$ $\mu \mathrm{m}^{2}$. Zoomed, changed in colour and enhanced in contrast details (A-D) are also shown. Inset (a) is also an image in negative colour with respect to the original one.

Figure 7 ( $\mathrm{a}$ and b) show an in vivo X-ray image of Leptolyngbya cells stored in an LiF crystal detectors and observed by the optical microscope in fluorescence mode. It is well visible the typical field of view of the holder, that is limited by the dimensions $\left(250 \times 250 \mu \mathrm{m}^{2}\right)$ of the silicon nitride window, which separates the soluble suspension of the cells from the vacuum chamber of soft X-ray source. It is important to stress that it would not be possible to acquire the whole field of view (all together, in a single image) by AFM on PMMA detectors because of the reading instrument typical characteristics. Close to the borders of the soft X-ray exposed area, the CCs photoluminescence is higher (brighter areas) just because of the enhanced water thickness at the centre of the $\mathrm{Si}_{3} \mathrm{~N}_{4}$ window, which is deformed by the atmospheric pressure of the water. Both images have been taken with the Leica microscope, equipped with a 16 bits Andor camera, at different magnifications.

Another example of the same biological sample is given in Figure 8. In this case the microscope was equipped with a 8-bit colour JVC camera, so that the typical yellow fluorescence light emitted by CCs in LiF is observable. The disadvantage of the 8 bits colour CCD camera is that the CCD dynamic range is so poor that no further information can be extracted from the fluorescence image stored in the LiF detector (as better explained later). Both in Figures 7 and 8 the shadow of the Leptolyngbya filaments are visible together with empty envelopes, which shows some rigidity points (black arrows in Fig. 7). Individual 


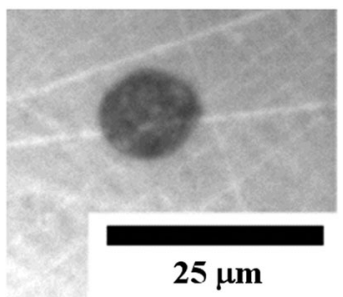

(A)

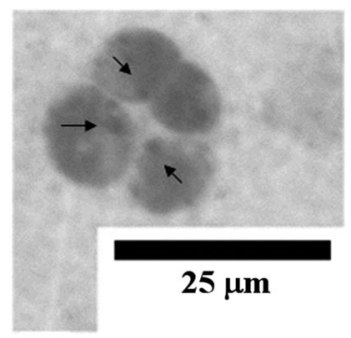

(B)

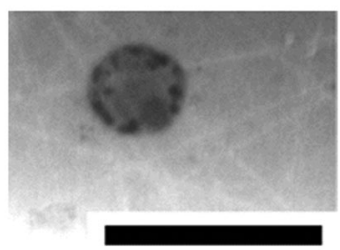

$25 \mu \mathrm{m}$

Fig. 6. X-ray images in the water-window of in vivo C. reinhardtii cells stored in a LiF crystal (exposed at the Nd-YAG laser plasma source) and observed by a fluorescence microscope. The autospores formation is shown (b).

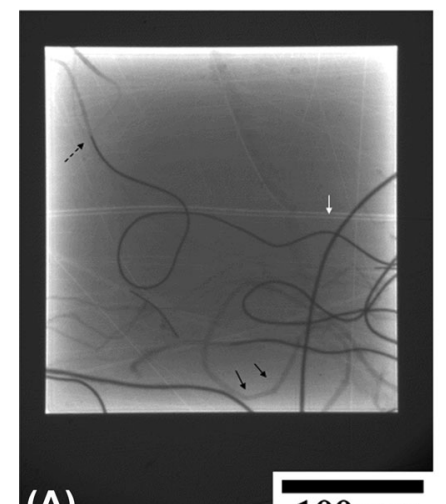

(A)

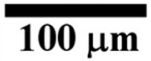

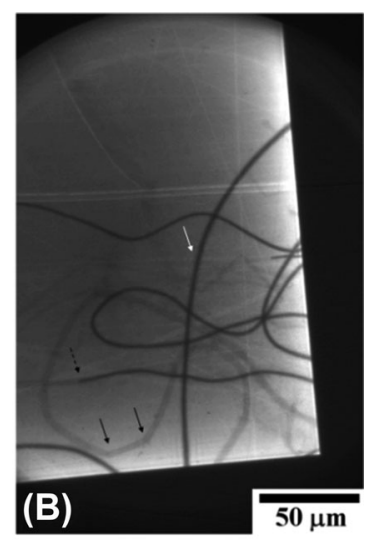

Fig. 7. X-ray images in the water-window of in vivo Leptolyngbya cells stored in a LiF crystal and observed by a Leica fluorescence microscope equipped with a black and white 16 bits Andor CCD camera using (A) a $50 \times$ objective or (B) a $100 \times$ objective. In (a) the full-view region of the $\mathrm{LiF}$ crystal is acquired.

cells forming the trichomes are not recognizable even if the presence of the filament is very well evidenced (white arrow in Fig. 7). One trichome apex is however noticeable (dashed black arrow in Fig. 7). It is therefore possible to recognize the end of filament and the beginning of the empty sheath.

In Figure 9(A), a detail of Figure 8, is observed at higher magnification using the 16 bits Andor CCD camera rather than the 8 bits colour one.

This zooming of the Leptolyngbya SXCM on LiF detector shows very well the sheath (white arrows), around $0.6 \mu \mathrm{m}$ thick, whereas the diameter of the filament is around $2.5 \mu \mathrm{m}$. The apical cell of the trichome (black arrow) clearly shows the

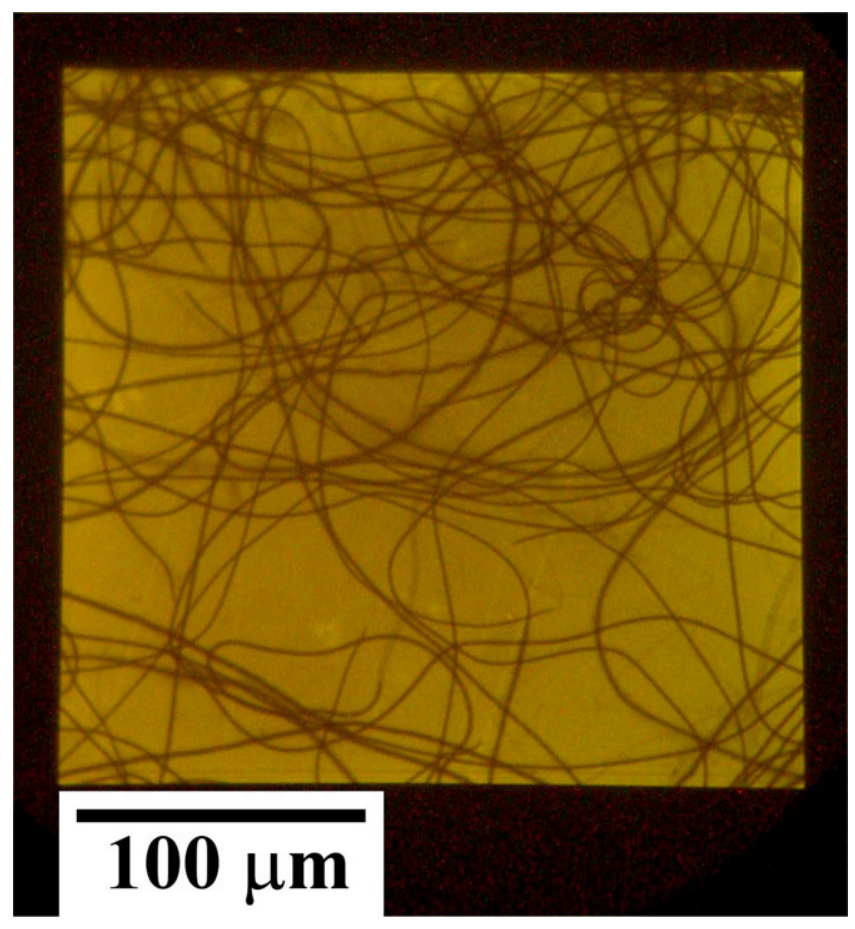

Fig. 8. Full-field image of a LiF crystal observed by a 8-bit colour CCD camera with a $50 \times$ objective of Leica microscope used in fluorescence mode: the darker fluorescence of LiF reveals the images of many filaments of leptolyngbya. The yellow colour of the crystal (in the $250 \times 250 \mu \mathrm{m}^{2}$ exposed region) is given by the combination of the red and of the green fluorescence light emitted by the $\mathrm{F}_{2}$ and $\mathrm{F}_{3}{ }^{+} \mathrm{CCs}$, respectively.

presence of a conical tip that is characteristic of this species. By increasing the contrast, see Figure 9(B), it is possible to recognize on the sheath the marks of the cells that it contained (this is not observable directly at optical microscope), but still nothing inside the filaments of cells even at exaggerated contrast (Fig. 9C), unless a special image reconstruction procedure is applied, as described in the following.

As already mentioned before, the spatial resolution of SXCM on LiF is mainly limited during the reading process with the optical fluorescence microscope because of the Abbe diffraction at visible wavelengths. This limit has particularly bad consequences in the case of leptolyngbya samples, because they are slightly transparent and so thin that the bright fluorescence light emitted by the strongly exposed LiF surrounding regions of the leptolyngbya filaments invades the region that corresponds to the filament. This effect generates an overlapping of nonuniform stray-light over the cells image (more intense close to the borders of the filament), thus preventing the possibility to recognize any internal structure of the cells. This degradation of the image cannot be recovered simply by a contrast/brightness adjustment (see Figs. 9B and C), because the stray light modulation inside the leptolyngbya region exceeds that of the true image, which could be observable by an ideal optical microscope. 

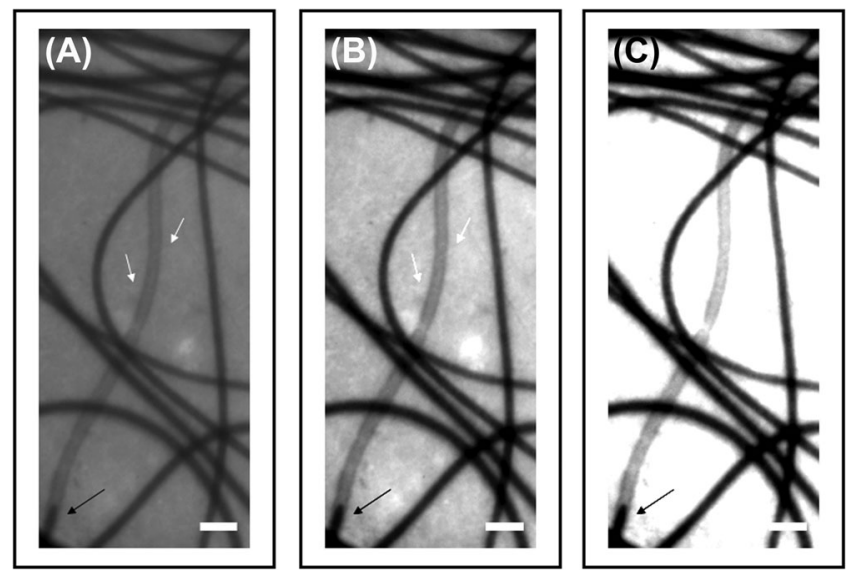

Fig. 9. Magnificated fluorescence image of a region of Figure 8(A) obtained by observing the LiF crystal with a $100 \times$ objective of Leica optical microscope equipped with a 16 bits Andor CCD camera. The white bar indicates $5 \mu \mathrm{m}$ spatial reference; (B) like (a) but at increased contrast; (C) like (b) with a further contrast enhancement.

The phenomena can be better understood by imaging, with the same microscope and same objective lens, a well-known object (similar to the filament shape of leptolyngbya) like, for example, a periodic reflective aluminium pattern $(5 \mu \mathrm{m}$ half period) deposited on a glass substrate by lithographic techniques, as shown in Figure 10, that is with very sharp borders of the patterns; the bright zones due to aluminium reflection (Fig. 10A) generate stray light that invade the dark regions, corresponding to the uncoated glass surface. The invasion occurs by more than $2 \mu \mathrm{m}$ (which is about 5 times the microscope resolution and it is also larger than a typical leptolyngbya cell diameter), thus preventing the possibility to observe any defect or impurities on the glass surface in such dark regions (like for the leptolyngbya case).

To partly reconstruct the original shape of the patterns, first of all we can generate a binary (only full white and full black grey levels) mask by assigning value 0 or 255 (for an 8 bits image) to all pixels which are below or over, respectively, a threshold selected as an intermediate grey level between those of the bright and of the dark regions of the original image (Fig. 10B). Then we can reduce the mask intensity to approximately $30 \%$ of the threshold (for example) and defocus it by applying a Gaussian blurring with a blurring radius similar to that of the optical microscope resolution (Fig. 10C). Most of the imaging software available on the market include the Gaussian blurring function, it is simply given by the following equation:

$\operatorname{Mask}^{\prime}(x, y)=\frac{\int_{-2 R b}^{2 R b} \int_{-2 R b}^{2 R b} \operatorname{Mask}(x, y) \times e^{-\frac{\left(x-x^{\prime}\right)^{2}+\left(y-y^{\prime}\right)^{2}}{R b^{2}}} d x^{\prime} d y^{\prime}}{\int_{-2 R b}^{2 R b} \int_{-2 R b}^{2 R b} e^{-\frac{\left(x-x^{\prime}\right)^{2}+\left(y-y^{\prime}\right)^{2}}{R b^{2}}} d x^{\prime} d y^{\prime}}$,
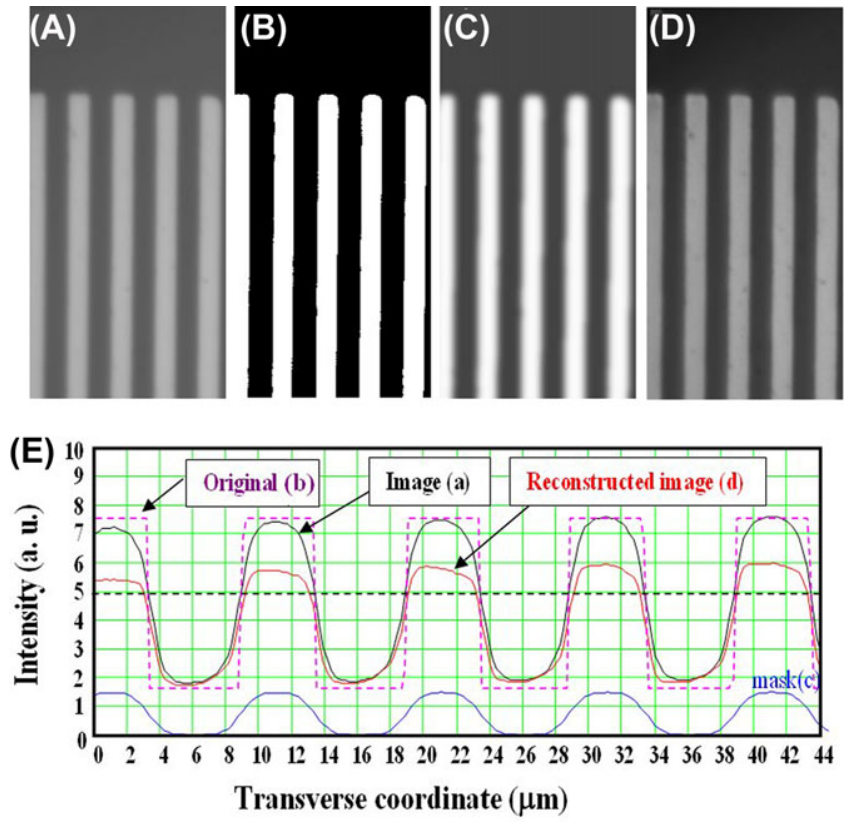

Fig. 10. (A) Periodic aluminium patterns (100 lines per $\mathrm{mm}$ ) on a glass substrate imaged by Leica microscope in reflection mode with a $100 \times$ objective; (B) binary mask extracted from (a) for a threshold intermediate between bright and dark regions grey levels; (C) same mask after a Gaussian blurring with $1 \mu$ m radius; (D) reconstructed image obtained by subtracting the defocused-mask " $c$ " to the original image "a" (in this case the mask intensity has been set at $30 \%$ of the threshold amplitude); (E) Horizontal intensity profile of the different steps of image reconstruction shown in a-d. In this last picture the image given by an ideal microscope corresponding to the original aluminium patterns is also reported (dashed curve); it coincides with the binary mask.

where $\operatorname{Mask}(x, y)$ is the binary mask, Mask' $(x, y)$ is the blurred mask and $R b$ is the blurring radius.

Finally, we can subtract the defocused mask to the original image in order to obtain the reconstructed image (Fig. 10D). As it can be seen, in this reconstructed image the borders of the bright region appear sharper (i.e. more similar to the original object) and the amplitude of the stray light in the dark regions is significantly flattened, so that now some details could be observed even in such regions (e.g. by increasing the contrast).

The improved flatness in dark regions can be better recognized by plotting the profiles of Figure 10(A-D) as shown in Figure 10(E). In this plot, beside the intensity profiles of Figures $10(\mathrm{~A}-\mathrm{D})$ is reported also the true shape of the aluminium patterns, that is the intensity profile that the image would have in case of an ideal microscope (aberration free).

The same reconstruction procedure was applied to the leptolyngbya image of Figure 9(A). The result is shown in Figure 11(A-D), where Figure 11(A) is identical to Figure 9(A), and it is reported again just for convenience. After the image reconstruction (Fig. 11D), internal details can be observed in the regions of the leptolyngbya filaments and the cells can be distinguished. As it can be seen in the intensity profiles 
(A)
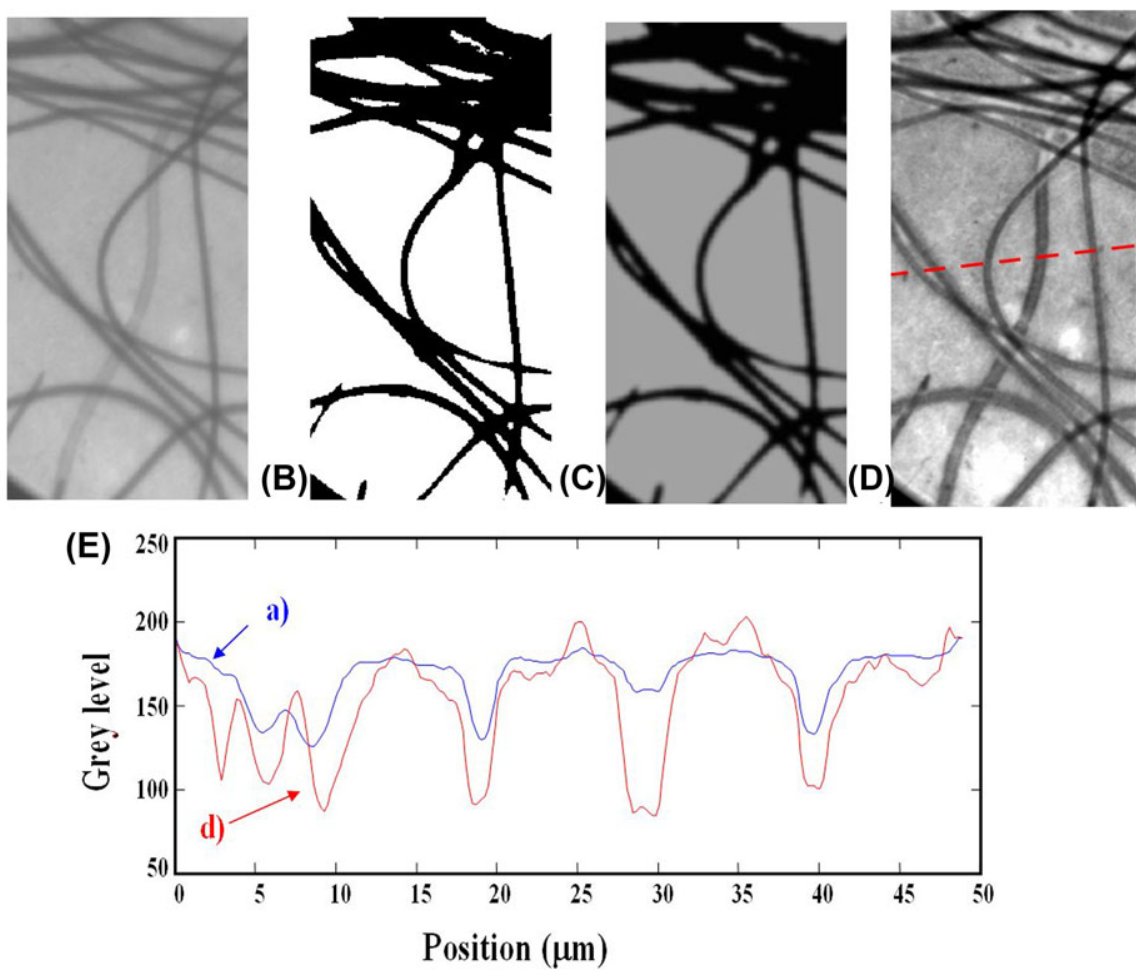

Fig. 11. Image reconstruction steps (like for Fig. 10) of Figure 9(A): (A) the original image; (B) the binary mask; (C) the defocused mask; (D) the reconstructed image; and finally (E) the profile comparison of original "a" and reconstructed "d" images along the red dashed line of the reconstructed image as shown in Figure 9(D).

comparison along a selected direction (Fig. 11E), the tubular morphology (double peak profile) of the leptolyngbya filaments, which was obscured by stray light in the original image, has been obtained after reconstruction (see in particular the filament at the $40 \mu \mathrm{m}$ position).

An even better contrast can be obtained by reversing to negative the reconstructed image, as shown in Figure 12 (where a bit wider region is considered). It is important to underline that such image reconstruction is possible just because of the wide dynamic range of luminescent LiF detector and of the used CCD camera (Andor 16 bits). Without such high dynamic, a much poorer contrast would be possible.

The observation of the cellular exudate, very clear in Figure 12, given by the high absorption contrast in the waterwindow soft X-rays, is rather difficult to obtain with other microscopy techniques (Bonfigli et al., 2008).

Figure 13 shows the $\mathrm{X}$ image of in vitro macrophage cells, line $R A W 264.7$, from mouse lung, stored in a LiF crystal and observed by the Leica microscope in fluorescence mode. It is obtained by the exposure of the cells directly in their medium, in the native state. The shadow of the cells on the LiF crystal surface, highlighted in the negative false colour in the image Figure 13(B), clearly show the circular shape of the cell and the presence of grains, possibly fagosomes. In the images the presence of the cytoplasm and of possible cell organelles is also

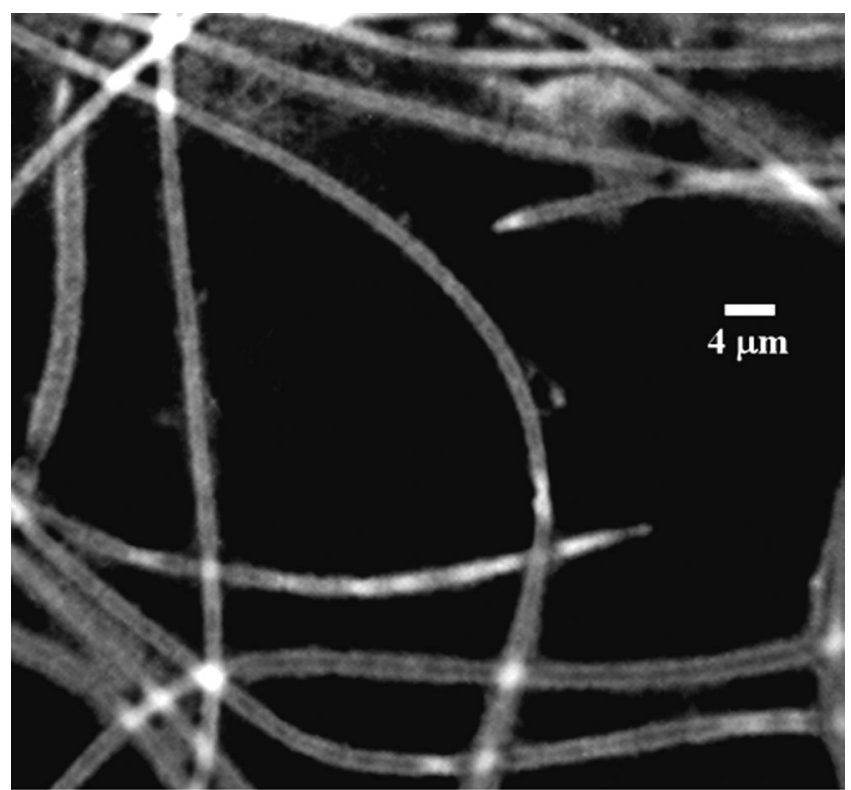

Fig. 12. Negative of the reconstructed image from Figure 9(A) (and neighbouring region). Two ends of leptolyngbya filaments are observable in this image. Some exudate from the filaments is also observable, more abundant where filaments group together. 

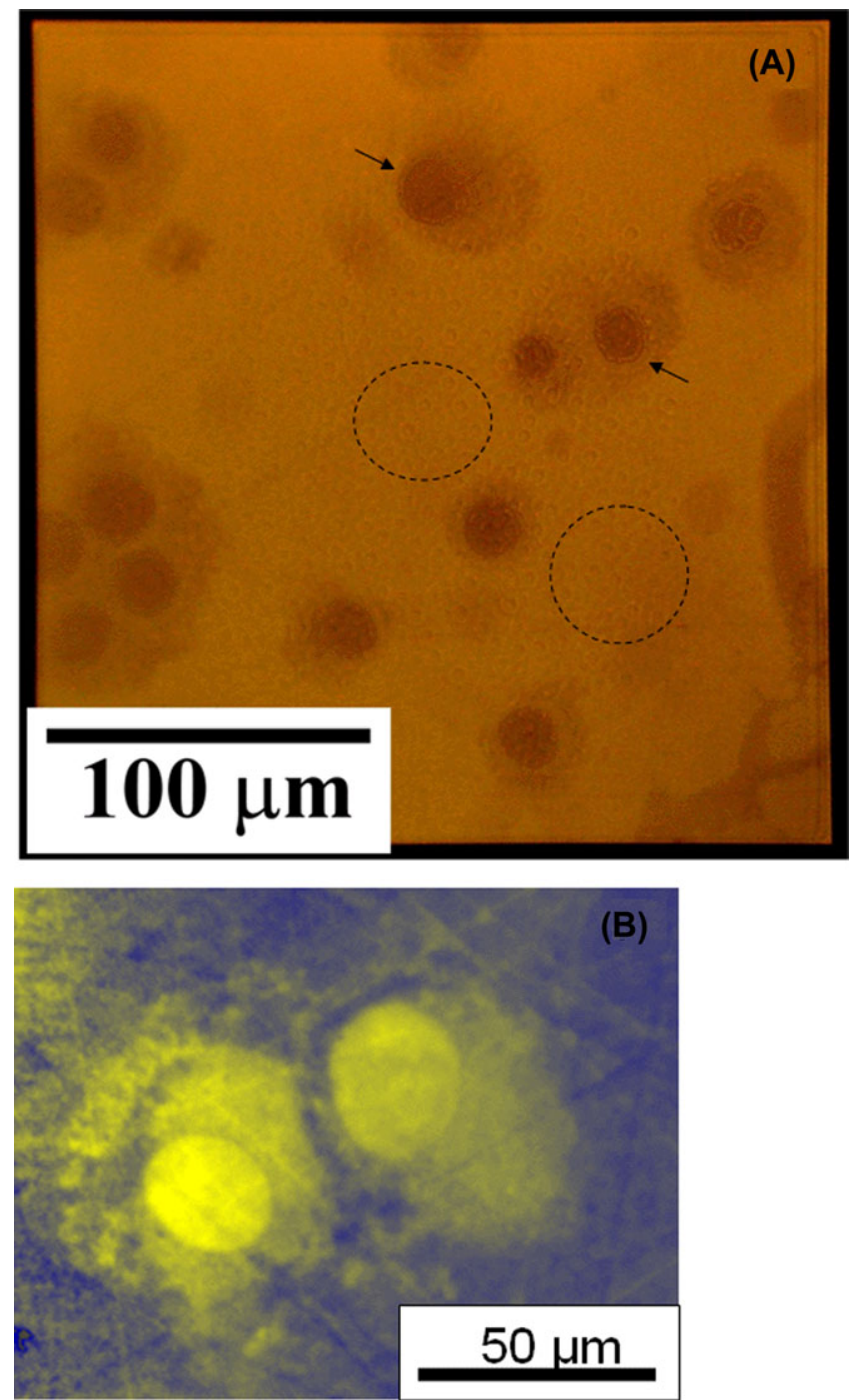

Fig. 13. X-ray images in the water-window of in vitro macrophage cells RAW 264.7 from mouse lung stored in a LiF crystal and observed by Leica optical microscope in fluorescence mode with (A) $50 \times$ objective or (B) $100 \times$ objective (reversed to negative for a better contrast enhancement).

recognizable as the presence of clouds surrounding the more intense shadow of the nucleus. The presence of a possible nuclear membrane is also visible (black arrows in Fig. 13A). The surface of the crystal evidences some damages (dashed circles in Fig. 13A) because of the soluble action of the liquid medium. This result shows that the protection effect of the benzene fat is important but still insufficient.

Figure 13(B) shows very well the shape of the nucleolus while the surrounding cytoplasm is not so well visible. It seems that the shadow of the cytoplasm is incomplete and fragmented; even in this case the reason could be the soluble action of the cell and water suspension of the medium where the cell were grown, which probably removed part of the image. For this reason not many details are recognizable, a part from the nucleolus and the weak shadow of the cytoplasm. Anyway from this image it is very well recognizable the size of the cells, which ranges between 20 and $25 \mu \mathrm{m}$.

\section{Conclusions}

In this paper, a morphology study of different kinds of cells is presented. It is one of the first attempts to analyse plant and animal cells by SXCM in the water-window energy range using LiF crystal as innovative solid-state imaging detector. Although the technique used here to protect the LiF surface from the cell solution medium needs to be further improved (the fat from benzene is not sufficiently uniform), the main characteristics of different biological samples have been successfully observed.

We showed that the peculiarities of this detector (wide dynamic range, very high spatial resolution and high saturation doses) are particularly suitable for SXCM of in vivo biological samples. In particular, we have here demonstrated that the high dynamic range of LiF allows revealing details even for cases (i.e. the leptolyngbya) in which the morphology of the biological sample has very high aspect ratios so that a strong stray light can be generated by fluorescence during the optical reading process of LiF detector by conventional microscope. The reconstruction technique for stray light removal here shown might be further improved by more sophisticated mathematical algorithms to achieve an even better image elaboration.

It was possible to evidence some cell structures of the cyanobacterium Leptolyngbya, two species of Chlamydomonas and the macrophage line RAW 264.7. All these samples are interesting to be analysed with the X-ray technique, which also introduces the possibility for an elemental analyses. Much effort could be done to observe these samples in different experimental conditions and to verify the possibility to localize pollutants at subcellular level. In particular, in the case of the macrophage line RAW 264.7 samples, it might be very interesting to study by SXCM this cell line after the growing in the presence of nano-particles.

\section{Acknowledgements}

Authors thank Prof. Armando Reale for his continuous support and precious suggestions on this research activity. The work was partially supported by RIA grants of University of L'Aquila to A. Poma. Work of T. Pikuz and A. Faenov were partly supported by Russian Science Foundation, grant \# 1450-00124.

\section{References}

Ackermann, W., Asova, G., Ayvazyan, V. et al. (2007) Operation of a free-electron laser from the extreme ultraviolet to the water window. Nat. Photon. 1, 336-342. 
Albertano, P.\& Grilli Caiola, M. (1988a) Effects of different light conditions on Lyngbya sp. in culture. Arch. Hydrobiol., Algol. Stud. 50-53, 47-54.

Albertano, P. \& Grilli Caiola M. (1988b) Structural and ultrastructural characters of a red biodeteriorating Lyngbya sp. in culture. Arch. Hydrobiol., Algol. Stud. 50-53, 55-57.

Albertano, P. (1991) Effects of monochromatic lights on four species of Leptolyngbya. Arch. Hydrobiol., Algol. Stud. 64, 199-214.

Albertano, P., Barsanti, L., Passarelli, V., \& Gualtieri, P. (2000) A complex photoreceptive structure in the cyanobacterium Leptolyngbya sp., Micron 31, 27-34.

Albertano, P., Reale, L., Palladino, L., et al. (1997) X-ray contact microscopy using an excimer laser plasma source with different target materials and laser pulse duration. J. Microsc. 187, 96-103.

Albertano, P., Belli, M., Bollanti, S., et al. (1998) Soft X-ray plasma source for atmospheric pressure microscopy, radiobiology and other applications. Nuovo Cimento D 20, 1685-1701.

Almaviva, S., Bonfigli, F., Franzini, I., Lai, A., Montereali, R.M., Pelliccia, D., Cedola, A., Lagomarsino, S. (2006) Hard x-ray contact microscopy with $250 \mathrm{~nm}$ spatial resolution using a LiF film detector and table-top microsource, Applied Physics Letters 89, 054102-4.

Athanasou, N.A. (1995) Synovial macrophages. Ann. Rheum. Dis. 54, 392-394.

Baldacchini, G., Bollanti, S., Bonfigli, F., et al. (2005), A novel soft X-ray submicron imaging detector based on point defects in LiF. Rev. Sci. Instrum. 76, 1113104-1-12.

Banchereau, J. \& Steinman, R.M. (1998) Dendritic cells and the control of immunity. Nature 392, 245-252.

Bellezza, S., Paradossi, G., De Philippis, R., \& Albertano, P. (2003) Leptolyngbya strains from Roman hypogea: cytochemical and physico-chemical characterisation of exopolysaccharides. J. Appl. Phycol. 15, 193-200.

Bold, H.C. (1949). The morphology of Chlamydomonas chlamydogama sp. nov. Bull. Torrey Bot. Club. 76, 101-108.

Bellucci I., Ciuffa P., Flora F., Martellucci S. \& Petrocelli G. (2000). Spettroscopia ad alta risoluzione di un plasma di Mg. Proc. "40 Anni di Laser (Firenze 2000)” Collana di quaderni di ottica e fotonica N. 7. Società Italiana di ottica e fotonica, 159-162.

Bonfigli, F., Baldacchini, G., Faenov, A., Flora, F., Marolo, T., Montereali, R.M., Nichelatti, E. \& Pikuz, T. (2003) Optical emitters based on color centers in lithium fluoride exposed to soft X-ray radiation (ed. by A. Pierre and R.B. Jr Fred), pp. 194-202. In Proceedings of the SPIE 5181 (2003) Wave Optics and Photonic Devices for Optical Information Processing II, SPIE, Bellingham, WA.

Bonfigli, F., Faenov, Y.A., Flora, F., et al. (2005) Point defects in lithium fluoride for micro-radiography. X-ray microscopy and photonic applications. Phys. Stat. Sol. (a) 202, 250-255.

Bonfigli, F., Faenov, A., Flora, F., et al. (2008) High-resolution water window X-ray Imaging of in vivo cells and their products using LiF crystal detectors. Microsc. Res. Tech. 71(1), 35-41.

Bonfigli, F., Flora, F., Franzini, I., Nichelatti, E. \& Montereali, R.M. (2009) Optical characterization of a soft X-ray imaging detector based on photoluminescent point defects in lithium fluoride thin layers. J. Lumin. 129, 1964-1967.

Bruno, L. \& Albertano, P. (1999) Photoacclimation of sciaphilous epilithic cyanobacteria isolated from Roman hypogea. Arch. Hydrobiol. Algol. Stud. 94, 89-103.

Chao, W., Harteneck, B.H., Liddle, J.A., Anderson, E.H. \& Attwood, D.T. (2005) Soft X-ray microscopy at a spatial resolution better than $15 \mathrm{~nm}$. Nature 435, 1210-1213.
Chapman, H.N., Barty, A., Bogan, M.J. et al. (2006) Femtosecond diffractive imaging with a soft-X-ray free-electron laser. Nat. Phys. 2, 839843.

Cotton, R., Bollanti, S., Di Lazzaro, P. et al. (1995) X-ray contact microscopy using a plasma source generated by long and short (120 ns and $10 \mathrm{~ns}$ ) excimer laser pulses. Proc. SPIE 2523 Applications of laser plasma radiation II (ed. by M. Richardson \& G. Kyrala). 184-193.

Hume, D.A. \& Gordon, S. (1983) Mononuclear phagocyte system of the mouse defined by immunohistochemical localization of antigen $\mathrm{F} 4 / 80$. Identification of resident macrophages in renal medullary and cortical interstitium and the juxtaglomerular complex. J. Exp. Med. 157, 1704 1709.

Hume, D.A., Halpin, D., Charlton, H. \& Gordon, S. (1984a) The mononuclear phagocyte system of the mouse defined by immunohistochemical localization of antigen F4/80: macrophages of endocrine organs. Proc. Natl. Acad. Sci. U. S. A. 81, 4174-4177.

Hume, D.A., Loutit, J.F. \& Gordon, S. (1984b). The mononuclear phagocyte system of the mouse defined by immunohistochemical localization of antigen F4/80: macrophages of bone and associated connective tissue. J. Cell. Sci. 66, 189-194.

Hume, D.A., Allan, W., Hogan, P.G. \& Doe, W.F. (1987) Immunohistochemical characterisation of macrophages in human liver and gastrointestinal tract: expression of CD4, HLA-DR, OKM1, and the mature macrophage marker 25F9 in normal and diseased tissue. J. Leukoc. Biol. $42,474-484$.

Larabell, C.A. and Le Gros, M.A. (2004) X-ray tomography generates 3-D reconstructions of the yeast, saccharomyces cerevisiae, at 60-nm resolution. Mol Biol Cell 15(3), 957-62.

Larsen, E.C., Ueyama, T., Brannock, P.M., et al. (2002) A role for PKC $\varepsilon$ in Fc $\gamma$ R-mediated phagocytosis by RAW 264.7 cells. J. Cell Biol. 159(6), 939-944.

Laskin, D.L., Weinberger, B. \& Laskin, J.D. (2001) Functional heterogeneity in liver and lung macrophages. J. Leukoc. Biol. 70, 163-170.

Lee, S.H., Starkey, P.M. \& Gordon, S. (1985) Quantitative analysis of total macrophage content in adult mouse tissues. Immunochemical studies with monoclonal antibody F4/80. J. Exp. Med. 161, 475-489.

Lohmann-Matthes, M.L., Steinmuller, C. \& Franke-Ullmann, G. (1994) Pulmonary macrophages. Eur. Respir. J. 7, 1678-1689.

Montereali, R.M. (2002) Point defects in thin insulating films of lithium fluoride for optical microsystems microsystems. Handbook of Thin Film Materials Vol. 3: Ferroelectric and Dielectric Thin Films. (ed. by H. S. Nalwa, San Diego CA, USA) Chap. 7, pp. 399-431. Academic Press.

Neutze, R., Wouts, R., van der Spoel, D., Weckert, E. \& Hajdu, J. (2000) Potential for biomolecular imaging with femtosecond X-ray pulse. Nature 406, 752-757.

Oldenhof, H, Zachleder, V. \& Van den Ende, H. (2006) Blue- and red-light regulation of the cell cycle in Chlamydomonas reinhardtii (Chlorophyta). Eur. J. Phycol. 41, 313-320.

Petrocelli G., Martellucci S. \& Richetta M. (1993a). Bismuth induced enhancement of the second-harmonic generation efficiency in bismuthsubstituted yttrium-iron garnet film. Appl. Phys. Lett. 63, 3402-3404.

Petrocelli G., Pichini E., Scudieri F. \& Martellucci S. (1993b). Anisotropic effects in the third-harmonic generation process in cubic crystals. JOSAB 10, 918-923.

Pickett-Heaps, J. (1975) Green Algae. Sinclair Associates, Inc., Sunderland, Massachusetts, pp. 27-57.

Reale, L., Bonfigli, F., Lai, A., et al. (2008) X-ray microscopy of plant cells by using LiF crystal as detector. Microsc. Res. Tech. 71, 839-848. 
Renaut, S., Replansky, T., Happlestone, A. \& Bell, G. (2006). The ecology and genetics of fitness in Chlamydomonas. XIII. Fitness of long-term sexual and asexual populations in benign environments. Evolution 60 , 2272-2279.

Richetta M., Francucci M., Gaudio P., et al. (2006) Soft X-ray generation by a tabletop Nd:YAG/GLASS laser system. J. Phys. Condens. Matter 18, 2039-2044.

Rippka, R., Deruelles, J., Waterbury, J.B., Herdman, M., \& Stanier, R.Y. (1979) Generic assignments, strain histories and properties of pure cultures of cyanobacteria. J. Gen. Microbiol. 111, 161.

Sakdinawat, A. \& Attwood, D. (2010) Nanoscale X-ray imaging. Nat. Photon. 4, 840-848.

Sekatskii, S.K. \& Letokhov, V.S. (1996) Single fluorescence centers on the tips of crystal needles: first observation and prospects for application in scanning one-atom fluorescence microscopy. Appl. Phys. B 63, 525530.

Ter-Mikirtychev, V.V. \& Tsuboi, T. 1997 On the nature of the $735 \mathrm{~nm}$ luminescence band in coloured LiF crystals. Can. J. Phys. 75(11), 813819.
Udagawa, N., Takahashi, N., Akatsu, T., et al. (1990) Origin of osteoclasts: mature monocytes and macrophages are capable of differentiating into osteoclasts under a suitable microenvironment prepared by bone marrow-derived stromal cells. Proc. Natl. Acad. Sci. U.S.A. 87, 7260-7264.

Ustione, A., Cricenti, A., Bonfigli, F., et al. (2006) Scanning nearfield optical microscopy images of microradiographs stored in lithium fluoride films with an optical resolution of $\lambda / 12$. Appl. Phys. Lett. 88, 141107-9.

Van Furth, R., Cohn, Z., Hirsh, J., Humprey, J., Spector, W., \& Langevoort, H. (1972) The mononuclear phagocyte system: a new classification of macrophage, monocyte and their precursor. Bull. WHO 46, 845-852.

Voitovich, A.P., Kalinov, V.S., Runets, L.P., Stupak, A.P., Martynovich, E.F., Montereali, R.M. \& Baldacchini, G. (2013) Color centers aggregation kinetics in lithium fluoride after gamma irradiation. J. Lumin. 143, 207-214.

Wachulak, P.W., Bartnik, A., Fiedorowicz, H. \& Kostecki J. (2011) A 50 $\mathrm{nm}$ spatial resolution EUV imaging-resolution dependence on object thickness and illumination bandwidth. Opt. Express 19(10), 95419550 . 